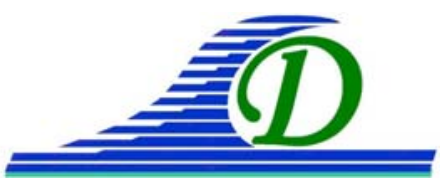
XIII ${ }^{\text {èmes }}$ Journées Nationales Génie Côtier - Génie Civil
Dunkerque, 2-4 juillet 2014

DOI:10.5150/jngcgc.2014.104 (C) Editions Paralia CFL

disponible en ligne - http://www.paralia.fr - available online

\title{
Démarche d'identification et valorisation des sédiments de barrage en matériaux routiers
}

\author{
Baptiste ANGER $^{1,2}$, Daniel LEVACHER ${ }^{1}$, Nitin MANGWAL ${ }^{3}$, François THERY $^{2}$ \\ 1. Université de Normandie, Unicaen, Laboratoire M2C, UMR CNRS 6143, 24 rue des \\ Tilleuls, 14000 Caen, France. \\ baptiste.anger@unicaen.fr \\ 2. EDF R\&D, Département Eco-efficacité et Procédés Industriels (EPI), site des \\ Renardières, 77818 Moret-sur-Loing. France. \\ 3. National Institute of Technology, Hamirpur, Himachal Pradesh 177005, India.
}

\section{Résumé :}

Le devenir des sédiments de dragage questionne de plus en plus les gestionnaires d'ouvrages et voies d'eau ainsi que les exploitants d'ouvrages hydroélectriques. La réglementation actuelle place clairement la gestion des sédiments à terre dans la nomenclature ICPE (Installation Classée Pour l'Environnement) et leur confère le statut de déchet. Cette problématique de gestion des sédiments amène à rechercher des solutions pérennes de valorisation. C’est dans ce cadre qu'une démarche a été entreprise par EDF SA. Parmi les voies potentielles où les sédiments fins pourraient être valorisés, la filière matériau de remblai ou de couche de forme routière a été très étudiée pour les sédiments marins. Peu d'études concernent par contre les sédiments de barrage.

Quatre types de sédiments provenant de retenues hydroélectriques françaises ont servi de support à une étude de valorisation matière. Une caractérisation géotechnique de base a minima, comprenant une analyse granulométrique, la détermination des limites d'Atterberg et les teneurs en $\mathrm{MO}$ et $\mathrm{CaCO}_{3}$ a été réalisée. Un traitement des sédiments à base de liants classiques à dosage économique a ensuite été mené. Deux formulations à base de chaux et de ciment ont été proposées pour des teneurs totales égales à $6 \%$ du poids du sédiment sec.

Les performances mécaniques des mélanges, en tenant compte du critère de traficabilité et du critère sensibilité à l'eau, ont été établies à partir d'essais de résistance en compression simple, en s’appuyant sur les normes en vigueur.

Mots clés : Sédiments de barrage, Caractérisation, Résistance, Durabilité, Valorisation.

\section{Introduction}

Les retenues hydroélectriques sont soumises à des dépôts de sédiments ayant un impact variable selon le bassin versant, la taille et la conception de la retenue. Pour des critères hydrauliques et d'efficacité de l'ouvrage ou d'usage du plan d'eau, il est nécessaire de gérer ces dépôts dans le respect de l'environnement et de la réglementation en donnant la priorité à la continuité sédimentaire. Dans certains cas, des impératifs d'exploitation 


\section{Thème 8 - Gestion et valorisation des sédiments marins}

ou environnementaux peuvent contraindre les opérateurs à s'écarter des solutions de gestion usuelles consistant à réintégrer les sédiments dans le cours d'eau, en aval de l'ouvrage, et envisager une gestion à terre. Si tel est le cas, les déblais de dragage i.e. les sédiments, destinés à une filière terrestre, entrent de fait dans la nomenclature déchets. Le décret du 13 avril 2010 relatif aux ICPE (JORF, 2010) et sa circulaire d'application du 24 décembre 2010 (BO, 2011), placent leur gestion à terre dans la nomenclature ICPE. De nouvelles rubriques pour les opérations de transit, de tri, de traitement et de stockage ont été mises en place. Certaines de ces opérations vont s'avérer difficiles à mettre en œuvre pour les sédiments de barrage.

En effet pour un site donné, la nature et le volume des dépôts va dépendre d'un certain nombre de facteurs tels que la géologie des sols du bassin versant, le régime climatique de la vallée, le régime hydrologique du cours d'eau, la couverture végétale du bassin versant, son degré d'activités humaines (pratiques culturales, assainissement urbain, industries...). Il en résulte de multiples typologies de sédiments et force est de constater qu'aucune démarche d'identification de sédiments de barrage pour une approche multifilière n’est proposée à ce jour.

\section{Démarche d'identification innovante}

Il n'existe pas de filière "robuste" de valorisation à terre des sédiments fins de barrage. Dans ce contexte complexe de gestion à terre, la connaissance des caractéristiques des sédiments est un élément essentiel pour les valoriser au mieux. Une des difficultés de leur valorisation est liée à l'absence de spécifications concernant les matériaux issus des dragages à l'entrée des filières et bien qu'il soit possible d'établir une comparaison avec les matériaux ayant des caractéristiques similaires traditionnellement utilisés, l'usage s’en trouve nettement plus limité.

Grâce aux récents travaux (COLIN, 2003 ; DUBOIS et al., 2009 ; KAMALI et al., 2008 ; MIRAOUI et al., 2012; TRAN, 2009; TRIBOUT, 2010), démontrant l'utilisation de matériaux issus de dragage possible comme matériau routier, on assiste à une amorce d'évolution dans cette filière, et on commence à reconnaître l'utilisation des sédiments comme possible.

Par exemple, le guide méthodologique pour l'utilisation de matériaux alternatifs en technique routière (SETRA, 2011) prévoit une application potentielle aux sédiments issus de dragage. De même, les sédiments sont présentés comme des gisements de matériaux dans la base OFRIR2 (2013) "Observatoire Français des Ressources pour les InfrastRuctures", développée par des organismes reconnus en matière d’infrastructures routières et de gestion des déchets. Ces matériaux restent cependant dans la catégorie des matériaux naturels "hors spécifications".

Les exploitants d'ouvrages, confrontés à la gestion des sédiments ont un réel besoin d'un outil d'aide à la décision quant aux filières potentielles de valorisation. En effet, il est important de constater qu'il n'existe pas de méthodologie de caractérisation des 


\section{XIII ${ }^{\text {èmes }}$ Journées Nationales Génie Côtier - Génie Civil \\ Dunkerque, 2-4 juillet 2014}

sédiments appropriée à leur valorisation prenant en compte plusieurs filières. Réaliser des études "mono-spécifiques", filière par filière, mène à des caractérisations démesurées, redondantes, coûteuses pour permettre d'aboutir finalement qu'à très peu d'applications pratiques.

Pour constituer les bases d'un tel outil, la démarche entreprise repose sur la proposition d'une caractérisation des sédiments avec un nombre réduit de paramètres en accord avec les spécifications techniques des filières potentielles envisageables. Pour ce faire, parmi les multiples réutilisations possibles des sédiments dragués, cinq filières majeures ont été pressenties. Elles correspondent à (i) une utilisation agronomique, (ii) une incorporation dans le matériau ciment et (iii) dans les bétons, (iv) une élaboration d'un matériau routier, et (v) une mise au point et conception d'un matériau céramique. Ces filières ont été choisies en considérant les éléments suivants :

- une faisabilité technique qui permet d'intégrer des sédiments fins,

- une capacité à absorber des volumes importants de sédiments,

- une mise en œuvre économiquement viable à faibles coûts,

- une adéquation avec la réglementation en vigueur,

- un accueil possible d'une ou plusieurs filières à proximité de la zone d'extraction des sédiments,

- une perspective d'être applicables ou extrapolables à d'autres voies de valorisation en utilisant des essais de caractérisation similaires.

Pour ces cinq filières étudiées, un travail de recherche des paramètres essentiels dans la caractérisation a été réalisé en s'appuyant sur la bibliographie existante (normes, guides techniques et articles scientifiques), sur des résultats et analyses d'essais en laboratoire et sur la considération d'avis d'experts. Cette démarche d'identification des paramètres d'entrée dans les filières de valorisation permet d'aboutir aux paramètres ayant une importance significative, c'est-à-dire aux paramètres-clés pour chacune des cinq voies de valorisation. Cela permet alors de tendre vers une caractérisation minimale des sédiments à valoriser (ANGER et al., 2012 \& 2013).

C'est dans cette démarche globale d'approche multi-filières visant à pré-orienter les sédiments que cet article s'intègre avec le cas d'une étude pour la valorisation par le traitement de sédiments fins de barrage comme "matériau routier et assimilé". Par "matériau routier et assimilé", il est entendu un usage potentiel comme couche de forme ou remblai routier dans une structure routière et dans des applications telles que des chemins piétonniers, des pistes cyclables, des aires de stationnement, ...

Pour cette étude, une caractérisation géotechnique simple mais suffisante des sédiments bruts est effectuée. Elle peut être employée pour caractériser l'ensemble d'un gisement et évaluer son homogénéité. Les performances mécaniques des sédiments fins de barrage traités aux liants hydrauliques sont ensuite évaluées. La résistance à la compression simple $\left(R_{C}\right)$ est utilisée comme indicateur de l'intégrité physique d'un matériau solidifié. 


\section{Les sédiments de barrage étudiés}

\subsection{Origine des sédiments}

Plusieurs lots de sédiments fins, issus de quatre retenues françaises exploitées par EDF ont été sélectionnés pour cette étude. Les sites échantillonnés ont été choisis de façon à refléter une certaine diversité typologique des sédiments, liée aux caractéristiques des bassins versants et des retenues. De plus, ces sites pourraient constituer des gisements de matériaux. Les quatre lots prélevés et étudiés ont été codifiés avec trois lettres : DUR, GAR, ISE et RHI.

\subsection{Caractérisation géotechnique de base}

La caractérisation géotechnique minimale comprend l'analyse granulométrique des sédiments, la mesure de leur teneur en matière organique et la détermination des teneurs en eau caractéristiques i.e. les limites d'Atterberg.

Ces trois paramètres sont en accord avec les différentes classifications géotechniques de référence qui permettent d'identifier les sols fins. Ils sont notamment fondamentaux dans le classement du GTR (SETRA \& LCPC, 1992). De plus, ces trois critères sont en accord avec les conclusions de DUAN (2008), qui indiquent qu'une caractérisation minimale, pour toute valorisation de sédiments, comprend la répartition dimensionnelle, les teneurs en matière organique et les limites d'Atterberg. Les caractéristiques des sédiments étudiés sont données dans le tableau 1. En complément de ces paramètres, la valeur au bleu des sédiments a été déterminée.

Tableau 1. Caractéristiques des sédiments étudiés.

\begin{tabular}{lllll}
\hline Sédiments & DUR & GAR & ISE & RHI \\
\hline Teneur en argile $(<2 \mu \mathrm{m})$ & 7,8 & 3,4 & 21,2 & 6,3 \\
Teneur en limon $(2$ à $63 \mu \mathrm{m})$ & 75,5 & 60,6 & 78,8 & 67,5 \\
Teneur en sable $(>63 \mu \mathrm{m})$ & 16,7 & 36,0 & - & 26,2 \\
Teneur en fines $(<80 \mu \mathrm{m})$ & 88,2 & 70,1 & 100 & 79,1 \\
Teneur en matière organique $(\%)$ & 3,0 & 9,1 & 3,6 & 6,9 \\
Limite de liquidité $-w_{L}(\%)$ & 39 & 76 & 46 & 71 \\
Limite de plasticité - $w_{P}(\%)$ & 30 & 50 & 30 & 46 \\
Indice de plasticité - $I_{P}(\%)$ & 9 & 26 & 16 & 25 \\
Valeur au bleu - VBS $(\mathrm{g} / \mathrm{100g})$ & 0,7 & 1,4 & 1,8 & 2,4 \\
\hline
\end{tabular}

\subsection{Classification des sédiments}

La caractérisation permet de classer ces sédiments selon plusieurs standards français et internationaux, voir tableau 2. Au-delà des classifications existantes, deux familles se distinguent clairement au regard de cette caractérisation. Une première avec des sédiments, DUR et ISE, peu plastiques, une très faible voire aucune fraction sableuse, et 


\section{XIII ${ }^{\text {èmes }}$ Journées Nationales Génie Côtier - Génie Civil \\ Dunkerque, 2-4 juillet 2014}

des teneurs en matières organiques faibles, proches de 3\%. La deuxième famille, avec les sédiments RHI et GAR, plus plastiques bien qu'avec une fraction sableuse plus importante et avec des teneurs en matières organiques plus significatives.

Dans le premier groupe, DUR et ISE, la faible teneur en matière organique ainsi que la faible plasticité sont des éléments favorables à leur utilisation. En revanche, la faible proportion en éléments grossiers sera à l'origine d'un squelette interne médiocre. Les sédiments du second groupe, GAR et RHI, qui sont à l'inverse plus sableux, présenteront une meilleure structure interne. Toutefois, la forte teneur en matière organique ainsi que la plasticité plus prononcée sont des éléments défavorables à l'efficacité du traitement et par conséquent, rendent difficile leur utilisation comme matériau routier.

Tableau 2. Classification des sédiments.

\begin{tabular}{llllll}
\hline Classement & Normes & DUR & GAR & ISE & RHI \\
\hline USCS & ASTM D 2487 (2011) & ML & OH & $M L$ & OH \\
AASHTO & AASHTO M-145 (2012) & $A-4$ & $A-7-5$ & $A-7-6$ & $A-7-5$ \\
GTR & GTR (SETRA \& LCPC, 1992) & $A_{1}$ & $F_{11}-A_{3}$ & $F_{11}-A_{2}$ & $F_{11}-A_{2}$ \\
\hline
\end{tabular}

\section{Moyens et méthodes pour une valorisation matériau de remblai router}

\subsection{Composition des mélanges et confection des éprouvettes}

Un traitement de stabilisation/solidification des sédiments à base de liants classiques à dosage économique a été mené. Deux formulations à base de chaux et de ciment ont été proposées pour des teneurs totales égales à $6 \%$ du poids du sédiment sec. Deux liants utilisés pour le traitement des sols fins, ont été retenus pour cette étude. Il s'agit d’un ciment Portland ordinaire de type CEM II B-M (S-LL) 32,5R et de la chaux vive en poudre $0 / 1,5 \mathrm{~mm}$, de teneur en $\mathrm{CaO}$ supérieure à $93 \%$.

Pour apprécier le dosage en chaux pour une stabilisation, EADES et GRIM (1966) ont développé l'essai de point de fixation de la chaux (PFC) qui permet de déterminer la quantité de chaux nécessaire et suffisante à apporter à un sol pour assurer à la fois une saturation des sites d'échanges des argiles et initier les réactions pouzzolaniques. En réalisant cet essai sur des sédiments, DUBOIS et al. (2009) et MAHERZI et al. (2012), ont observé qu'un pourcentage de chaux de $3 \%$ stabilise la valeur du $\mathrm{pH}$ à 12,4 et permet d'obtenir un milieu favorable au développement d'hydrates cimentaires et au développement de performances mécaniques. Un dosage similaire, avec $3 \%$ de chaux a donc été retenu dans une formulation.

Pour qu'une formulation soit dite "économique", le dosage en liants doit être inférieur à 7\% (FOUCHER, 2003). Pour respecter ce critère, deux formulations ont été définies. La première formulation, notée $\mathrm{A}$, est réalisée avec un dosage à hauteur de $3 \%$ de chaux et $3 \%$ de ciment. La deuxième, notée $\mathrm{B}$, avec du ciment seul à hauteur de $6 \%$ de la matière 
sèche des sédiments, voir tableau 3. Pour chacun des quatre sédiments, les deux formulations sont appliquées.

Tableau 3. Dosage en liants des mélanges.

\begin{tabular}{lll}
\hline Formulation & $\boldsymbol{A}$ & $\boldsymbol{B}$ \\
\hline Ciment (\%) & 3 & 6 \\
Chaux (\%) & 3 & - \\
\hline
\end{tabular}

Un protocole a été établi pour permettre d'assurer la répétabilité des opérations suivantes : séchage, malaxage, confection et conservation des échantillons. Dans un premier temps, les sédiments bruts sont séchés dans une étuve à $50{ }^{\circ} \mathrm{C}$ pour atteindre la teneur en eau fixée à 30\%. Cette teneur a été déterminée au regard de la teneur en eau à l'optimum Proctor que l'on rencontre pour ce type de sédiment subissant un traitement, généralement comprise entre 20 et 35\% (MAHER et al., 2004 ; JAUBERTHIE et al., 2010 ; SILITONGA, 2010 ; BEHMANESH, 2008 ; BOUTOUIL et al., 2011 ; COLIN, 2003 ; MIRAOUI, 2010 ; DERMATAS et al., 2003). Les sédiments de barrage sont ensuite mélangés aux liants dans un malaxeur durant 3 minutes.

Les éprouvettes sont ensuite moulées dans des étuis cylindriques en PVC ayant un diamètre intérieur de $34 \mathrm{~mm}$ et un élancement de 2. Le compactage est réalisé manuellement par piquetage tel que décrit par LIANG (2012). L'objectif de cette méthode rapide à mettre en oeuvre est de permettre un compactage à une énergie équivalente par volume, relativement proche de celle d'un compactage Proctor normal.

\subsection{Approche expérimentale et essais de validation mécanique et de durabilité}

L'approche expérimentale retenue dans cette étude, consiste à évaluer les performances mécaniques des sédiments traités en tenant compte de deux critères à satisfaire pour un matériau routier, à savoir, le critère de traficabilité complété du critère d'insensibilité à l'eau tels que définis dans le guide GTS (SETRA \& LCPC, 2000), voir tableau 4 ciaprès. Pour une étude complète, deux critères supplémentaires doivent être considérés : la résistance au gel et les performances escomptables à long terme.

Cinq séries d'éprouvettes pour chaque sédiment et formulation ont été compactées puis démoulées. Quatre de ces séries ont été conservées à l'air ambiant à $20{ }^{\circ} \mathrm{C} \pm 2{ }^{\circ} \mathrm{C}$ jusqu'à faire l'objet d'un essai de résistance à la compression simple $\left(R_{C}\right)$. Les essais ont été réalisés pour des temps de cure de 7, 14, 28 et 60 jours afin d'observer l'évolution des résistances mécaniques. Ce mode de conservation à l'air reproduit un milieu naturel moyennement favorable au développement des résistances mécaniques. En effet, l'action des liants n'est pas optimale dans ces conditions. Dans des situations réelles, il est donc possible d'atteindre de meilleures performances mécaniques. 


\section{XIII ${ }^{\text {èmes }}$ Journées Nationales Génie Côtier - Génie Civil \\ Dunkerque, 2-4 juillet 2014}

Tableau 4. Performances à atteindre après traitement, pour les paramètres retenus dans cette étude en vue d'une utilisation en couche de forme d'après le GTS (SETRA \& LCPC, 2000).

\begin{tabular}{lll}
\hline Critères à satisfaire & & Exigences mécaniques requises \\
\hline Critère de traficabilité & $R c$ & $\geq 1,0 \mathrm{MPa}$ \\
Critère d'insensibilité à l'eau & $R_{C I} / R_{C 60}$ & $\geq 0,8 \mathrm{si} \mathrm{VBS} \leq 0,5 ; \geq 0,6 \mathrm{si}$ VBS $>0,5$ \\
\hline
\end{tabular}

La cinquième série d'éprouvettes a permis d'évaluer la sensibilité à l'eau des mélanges. Dans cette optique, les éprouvettes ont été conservées 28 jours à l'air puis immergées durant 32 jours avant de faire l'objet d'un essai de compression simple (figure 1). La mesure est alors notée $R_{C I}$. La résistance à l'immersion au jeune âge étant définie par le rapport $R_{C I} / R_{C 60}$.

Les essais de compression simple ont été réalisés sur une presse à une vitesse de déformation imposée de $1,93 \mathrm{~mm} / \mathrm{min}$. La résistance à la compression simple est la moyenne des résultats obtenus avec trois éprouvettes. Avant essai, les extrémités des éprouvettes ont subi un léger surfaçage de manière à obtenir deux surfaces plates et parallèles.

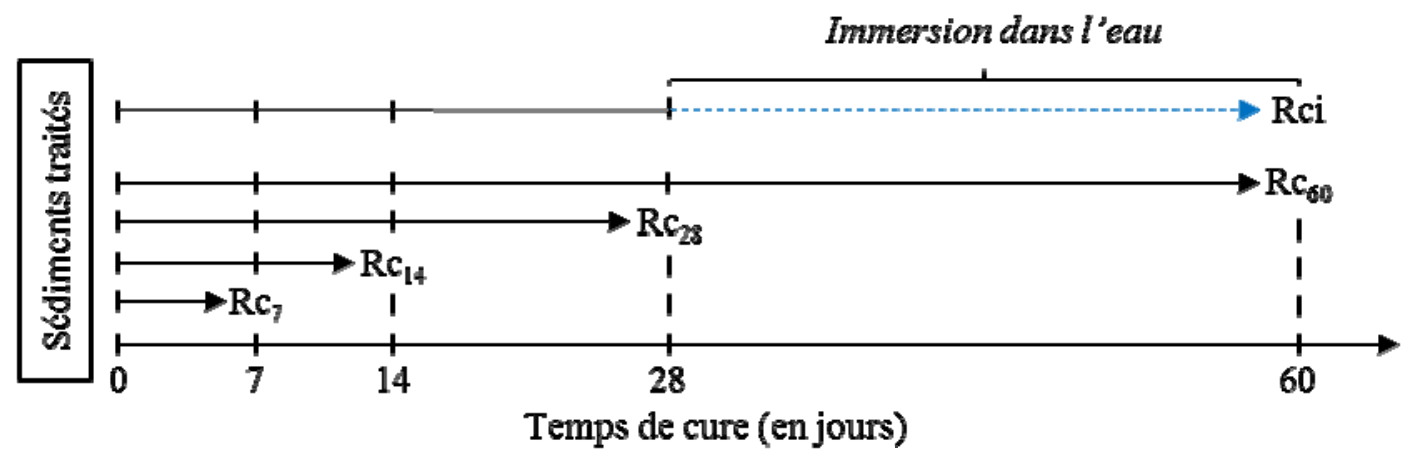

Figure 1. Organisation des essais et conservation des éprouvettes

\section{Résultats et discussion}

Pour une utilisation comme couche de forme, la résistance en compression doit être supérieure à $1 \mathrm{MPa}$ et le rapport $R_{C I} / R_{C 60}$ supérieur à 0,6 si l'argilosité est significative. Tous les sédiments étudiés ont une valeur au bleu de méthylène (VBS) supérieure à $0,5 \mathrm{~g}$ de bleu / $100 \mathrm{~g}$ de sol. Notons que dans le cas de voies piétonnes ou de pistes cyclables, il n'existe pas de guide technique officiel mais les sollicitations sur la structure sont moindres. Les exigences mécaniques seraient alors plus faibles. 


\subsection{Evolution des résistances à la compression}

Les évolutions temporelles des résistances mécaniques à la rupture obtenues pour les mélanges sont données sur la figure 2. Les barres d'incertitudes indiquent les valeurs minimales et maximales.

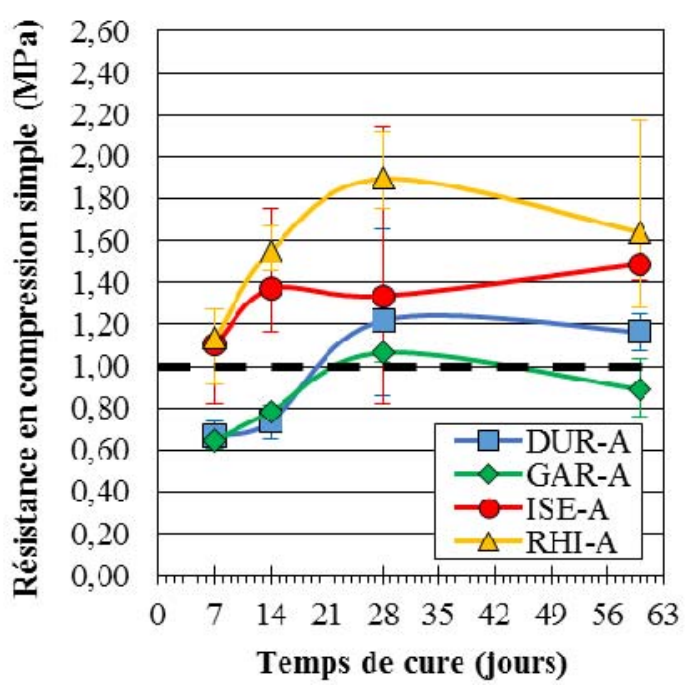

Formulation A (3\% chaux + 3\% ciment)

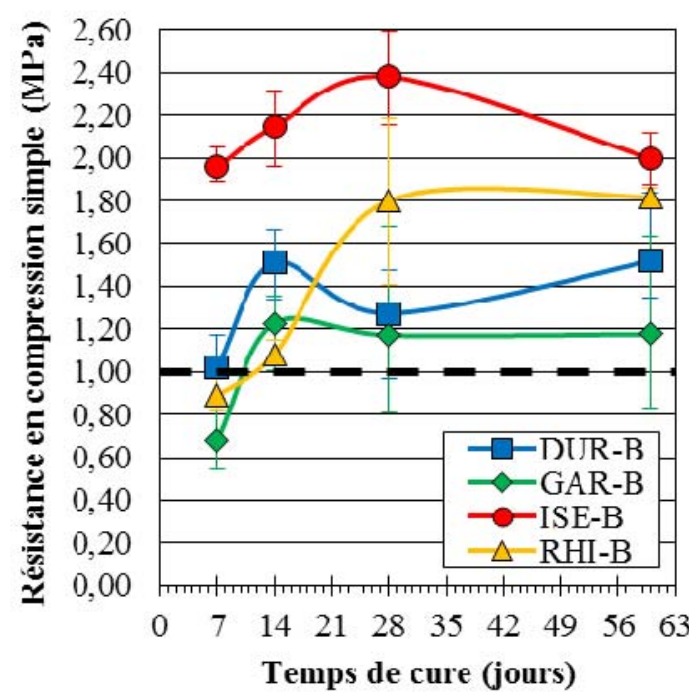

Formulation B (6\% ciment)

Figure 2. Evolution comparée des résistances en compression simple.

Pour la formulation A, les résistances augmentent jusqu'à 28 jours dans la plupart des cas. Au-delà de cette date, les mélanges marquent une légère diminution, excepté le sédiment ISE. L'étendue de résistance à 7 jours va de 0,64 à 1,13 $\mathrm{MPa}$, elle passe de 0,89 à $1,64 \mathrm{MPa}$ à 60 jours. Le seuil de $1 \mathrm{MPa}$ est dépassé pour tous les sédiments à 28 jours mais à 60 jours le sédiment GAR ne dépasse pas cette valeur.

Pour la formulation $\mathrm{B}$, le développement des résistances semble plus rapide. On constate qu'elles atteignent toutes des valeurs supérieures à $1 \mathrm{MPa}$, resistance seuil autorisant la circulation sur la couche traitée, dès 14 jours. L'intervalle de résistance obtenu à 7 jours se répartit entre 0,68 et 1,96 MPa. Le sédiment ISE développe à court terme des résistances mécaniques très satisfaisantes. A 60 jours les valeurs observées vont de 1,18 à 2,00 MPa. Les résistances mécaniques à 60 jours $\left(R_{C 60}\right)$ sont légèrement meilleures pour la formulation $\mathrm{B}$ avec un dosage à $6 \%$ de ciment que la formulation $\mathrm{A}$. Ceci est observé pour tous les sédiments.

\subsection{Résistance à l'immersion "au jeune âge"}

Afin d'apprécier l'influence du mode de cure sur la résistance en compression simple, des histogrammes comparatifs sont tracés sur la figure 3. 


\section{XIII ${ }^{\text {èmes }}$ Journées Nationales Génie Côtier - Génie Civil \\ Dunkerque, 2-4 juillet 2014}

Les résistances à l'immersion sont plus faibles pour les mélanges de composition A que pour les mélanges de composition $\mathrm{B}$. Les rapports $R_{C I} / R_{C 60}$, permettant de qualifier les mélanges au regard du critère d'insensibilité à l'eau, sont donnés dans le tableau 5.

Pour les mélanges de composition $\mathrm{A}$, les rapports $R_{C I} / R_{C 60}$ vont de 0,42 à 0,67 . Seul le sédiment DUR est au-dessus du ratio de 0,6. Pour les mélanges avec la composition $B$, les rapports $R_{C I} / R_{C 60}$ vont de 0,51 à 0,89 . Seul le sédiment ISE ne satisfait pas le critère d'insensibilité à l'eau pour les deux formulations. Cette observation est à relativiser par le fait que pour le mélange ISE-B, $R_{C I}$ atteint 1,01 MPa. Bien que le sédiment se montre sensible à l'eau, le niveau de résistance observé après immersion reste satisfaisant.

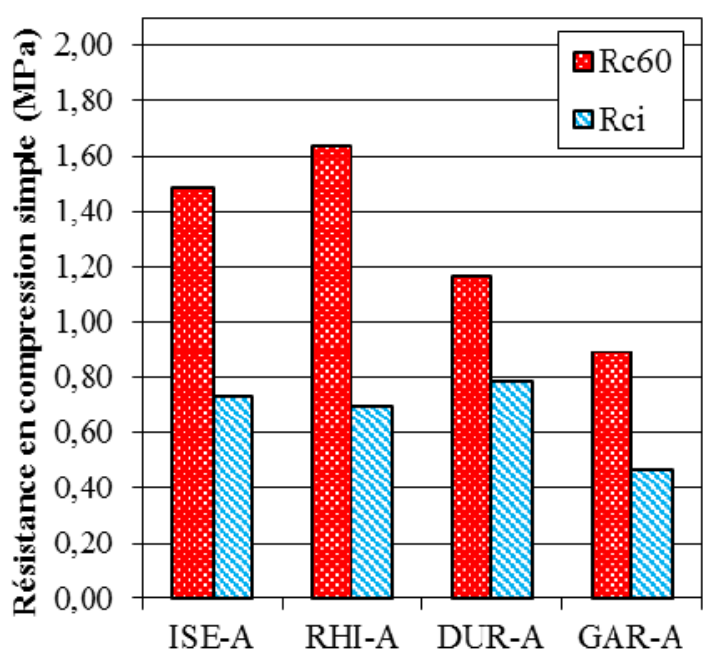

Formulation A (3\% chaux $+3 \%$ ciment)

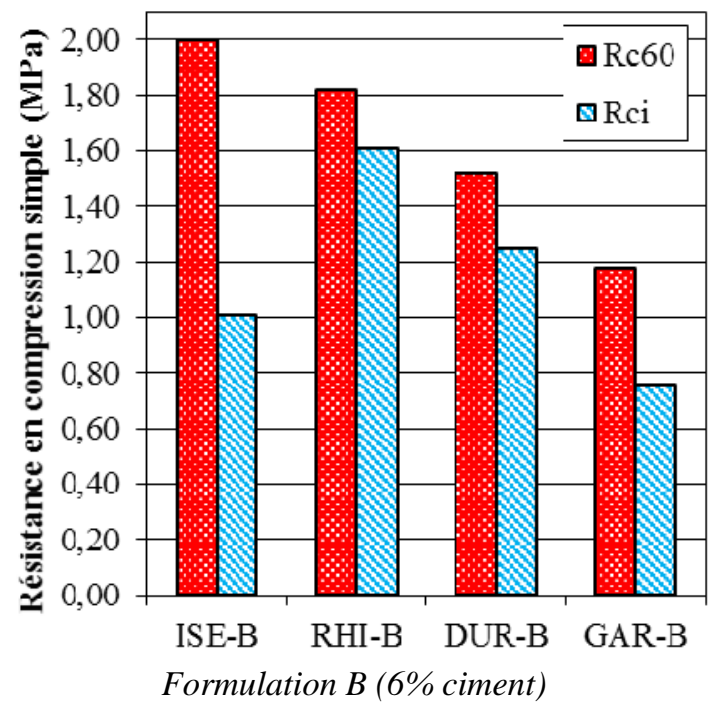

Formulation $\mathrm{B}$ (6\% ciment)

Figure 3. Valeurs comparées de $R_{C 60}$ et de $R_{C I}$.

Tableau 5. Résistance à l'immersion au jeune âge des mélanges.

\begin{tabular}{lllllllll}
\hline & ISE-A & RHI-A & DUR-A & GAR-A & ISE-B & RHI-B & DUR-B & GAR-B \\
\hline$R_{C I} / R_{C 60}$ & 0,49 & 0,42 & 0,67 & 0,53 & 0,51 & 0,89 & 0,82 & 0,65 \\
\hline
\end{tabular}

La finesse des grains du sédiment ISE peut être une hypothèse pour expliquer sa sensibilité à l'eau. Celui-ci présente un faible squelette interne. Il s'agit en effet du sédiment qui présente la granulométrie la plus fine, le seul qui soit dépourvu de fraction sableuse. Afin de limiter la sensibilité à l'eau, il est envisageable d'utiliser un correcteur granulométrique ou d'employer un ciment de classe de résistance supérieure.

Le sédiment DUR présente des résistances à l'immersion qui lui permettent de satisfaire le critère pour les deux formulations. Cependant, il se montre, comme pour les sédiments, moins sensible à l'eau avec la formulation $\mathrm{B}$. Ainsi, au regard de la sensibilité à l'eau, la formulation B est à préconiser. 


\section{Conclusion}

La procédure de caractérisation géotechnique a permis d’orienter le choix des compositions des mélanges pour une stabilisation aux liants classiques. Les sédiments sont en majorité des limons plus ou moins organiques. L'étude visait un réemploi des sédiments de barrage traités en matériau routier ou assimilé.

Cette étude a été réalisée à partir d’un niveau de caractérisation géotechnique adapté, complétée de l'analyse des performances mécaniques de deux formulations: l'une, notée A avec un dosage de 3\% de chaux et 3\% de ciment et la seconde, notée B, avec un dosage de $6 \%$ de ciment.

$\mathrm{Au}$ regard du critère de traficabilité, les résistances mécaniques des mélanges des compositions A (à l'exception du mélange GAR-A) et B satisfont la valeur seuil de $1 \mathrm{MPa}$. Ce critère est atteint dès 14 jours pour les sédiments traités pour la formulation B. Pour tous les sédiments étudiés, les résistances obtenues à 60 jours avec la formulation B sont meilleures que celles obtenues avec la formulation A.

$\mathrm{Au}$ regard du critère d'insensibilité à l'eau, la formulation A ne se montre pas suffisamment efficace. En revanche, la formulation B satisfait ce critère dans tous les cas à l'exception du sédiment ISE qui reste sensible à l'eau. Cependant, le niveau de résistance observé après immersion du mélange ISE-B, proche de $1 \mathrm{MPa}$, reste satisfaisant.

La formulation B, avec 6\% de ciment, est donc plus efficace que la formulation A pour l'ensemble des sédiments au regard des résistances mécaniques et ce pour les deux modes de conservation (cure à l'air et immersion). Cette formulation est préconisée dans le cas des sédiments de barrage étudiés. Les résultats obtenus sont encourageants pour envisager des valorisations matières des sédiments de barrage dans des applications géotechniques telles que des couches de forme, pistes cyclables, voies piétonnes, etc. Des études complémentaires restent nécessaires avant d'effectuer une réalisation. Elles porteraient notamment sur la caractérisation complète des gisements afin d'en évaluer l'homogénéité, les caractérisitiques de mise en œuvre et les performances escomptables à long terme à l'aide d'essais de traction indirecte et de la détermination des modules d'élasticité des sédiments traités.

Bien que le cadre actuel place les sédiments gérés à terre comme un déchet, ceux-ci peuvent être considérés comme une ressource d'origine naturelle et renouvelable donc pérenne pour sa valorisation dans le temps.

\section{Références bibliographiques}

AASHTO M-145 (2012). Classification of Soils and Soil-Aggregate Mixtures for Highway Construction Purposes. American of State Highway and Transportation Officials.

ANGER B., THERY F., LEVACHER D. (2012). Pour une caractérisation minimale des sédiments fins : application d'une démarche pour leur valorisation en matériaux 


\section{XIII ${ }^{\text {èmes }}$ Journées Nationales Génie Côtier - Génie Civil \\ Dunkerque, 2-4 juillet 2014}

routiers, XII ${ }^{\text {èmes }}$ Journées Nationales Génie Côtier - Génie Civil, 12-14 juin 2012, Cherbourg, France, pp 973-984. http://dx.doi.org/10.5150/jngcgc.2012.107-A

ANGER B., LEVACHER D., THERY F. (2013). A minimal characterization identification approach for pre-selecting dam fine sediments reuse option. 8th International SedNet conference, 6-9 November 2013, Lisbon, Portugal.

ASTM D 2487 (2011). Standard Practice for Classification of Soils for Engineering Purposes (Unified Soil Classification System). American Society for Testing and Materials.

BEHMANESH J. (2008). Etude de la durabilité d'un sédiment, traité au ciment et additifs. Thèse de doctorat, Université de Caen Basse-Normandie, 216 p.

BO (2011). Circulaire du 24/12/10 relative aux modalités d'application des décrets $n^{\circ}$ 2009-1341, 2010-369 et 2010-875 modifiant la nomenclature des installations classées exerçant une activité de traitement de déchets. BO du MEDDTL n ${ }^{\circ} 1$ du 25/01/2011.

BOUTOUIL M., BEN ABDELGHANI F., SAUSSAYE L., MAHERZI W. (2011). Étude du comportement des sédiments de dragage marins à l'essai d'aptitude au traitement. 2ème Conférence Méditerranéenne Côtière et Maritime, 22 - 24 novembre 2011, Tanger, Maroc, pp 141-144. http://dx.doi.org/10.5150/cmcm.2011.030

COLIN D. (2003). Valorisation de sédiments fins de dragage en technique routière. Thèse de doctorat, Université de Caen Basse-Normandie, 182 p.

DERMATAS D., DUTKO P., BALORDA-BARONE J., MOON D.H. (2003). Evaluation of engineering properties of cement treated Hudson River dredged sediments for reuse as fill materials. J. Marine Environ. Eng., Vol. 7, pp 101-123.

DUAN Z. (2008). Caractérisation, stabilisation et solidification de sédiments fins marins. Thèse de doctorat, Université de Caen Basse-Normandie, 145 p.

DUBOIS V., ABRIAK N.E., ZENTAR R., BALLIVY G. (2009). The use of marine sediments as a pavement base material. Waste Management, Vol. 29, pp 774-782. http://dx.doi.org/10.1016/j.wasman.2008.05.004

EADES J.L., GRIM R.E. (1966). A quick test to determine lime requirements for lime stabilization. Highway Research Record, pp 61-72.

FOUCHER J.M. (2003). Etude de valorisation des sédiments de dragage du chenal de la Seine en technique routière. Rapport DESS Gelipode, Université de Caen, 89 p.

JAUBERTHIE R., RENDELL F., RANGEARD D., MOLEZ L. (2010). Stabilisation of estuarine silt with lime and/or cement. Applied Clay Science, Vol. 50, pp 395-400. http://dx.doi.org/10.1016/j.clay.2010.09.004

JORF -Journal Officiel de la République Française- (2010). Décret n 2010-367 du 13 avril 2010 modifiant la nomenclature des installations classées et ouvrant certaines rubriques au régime de l'enregistrement. JORF n87 du 14 avril 2010.

KAMALI S., BERNARD F., ABRIAK N.E., DEGRUGILLIERS P. (2008). Marine dredged sediments as new materials resource for road construction. Waste Management, Vol. 28, pp 919-928. http://dx.doi.org/10.1016/i.wasman.2007.03.027 
LEVACHER D., SANCHEZ M. (2011). Caractérisation de sédiments marins pour une mise en dépôt à terre et en remblai. European Journal of Environmental and Civil engineering, Vol. 15(2), pp 167-178. http://dx.doi.org/10.3166/ejece.15.167-178

LEVACHER D., SANCHEZ M., DUAN Z., LIANG Y. (2011). Valorisation en unité pilote de sédiments méditerranéens : étude des caractéristiques géotechniques et de la perméabilité. Revue Paralia, Vol. 4, pp 4.1-4.20. http://dx.doi.org/10.5150/revue-paralia.2011.004 LIANG Y. (2012). Co-valorisation de sédiments et de sols fins par apport de liants et de fibres. Thèse de doctorat, Université de Caen Basse-Normandie, 194 p.

MAHER A., BENNERT T., JAFARI F., DOUGLAS W., GUCUNSKI N. (2004). Geotechnical properties of stabilized dredged material from New York-New Jersey Harbor. Transport. Research Record, Vol. 1874, pp 86-96. http://dx.doi.org/10.3141/1874-10

MAHERZI W., SAUSSAYE L., BOUTOUIL M. (2012). Aptitude au traitement et comportement mécanique des sédiments de dragage. XII ${ }^{\text {èmes }}$ Journées Nationales Génie Côtier - Génie Civil, 12-14 juin 2012, Cherbourg, France, pp 1059-1066. http://dx.doi.org/10.5150/jngcgc.2012.116-M

MIRAOUI M. (2010). Mise en oeuvre d'une démarche de prétraitement et de traitement des sédiments de dragage en vue d'une valorisation dans le génie civil. Thèse de doctorat, Ecole des Mines de Douai, $225 \mathrm{p}$.

MIRAOUI M., ZENTAR R., ABRIAK N.-E. (2012). Road material basis in dredged sediment and basic oxygen furnace steel slag. Construction and Building Materials, Vol. 30, pp 309-319. http://dx.doi.org/10.1016/j.conbuildmat.2011.11.032

SETRA, LCPC (1992). Guide Technique - Réalisation des remblais et des couches de forme, Fascicule 1, principes généraux. 100 p.

SETRA (2011). Acceptabilité de matériaux alternatifs en technique routière, Evaluation environnementale guide méthodologique. $32 \mathrm{p}$.

SETRA, LCPC (2000). Guide Technique - Traitement des sols à la chaux et/ou aux liants hydrauliques : application à la réalisation des remblais et des couches de forme. $240 \mathrm{p}$.

SILITONGA E. (2010). Valorisation des sédiments marins contaminés par solidification/stabilisation à base de liants hydrauliques et de fumée de silice. Thèse de doctorat, Université de Caen Basse-Normandie, 229 p.

TRAN N.T. (2009). Valorisation de sédiments marins et fluviaux en technique routière. Thèse de doctorat, Ecole des mines de Douai, 189 p.

TRIBOUT C. (2010). Valorisation de sédiments traités en techniques routières: contribution à la mise en place d'un protocole d'acceptabilité. Thèse de doctorat, INSA de Toulouse, $295 \mathrm{p}$.

OFRIR2 (2013). Catégories de matériaux - Sédiment de dragage, $<$ http://ofrir2.ifsttar.fr/materiaux/categories-de-materiaux/materiaux-naturelshspec/sediment-de-dragage/> (consulté le 09/12/2013) 